\title{
Digestibilidade e consumo dos nutrientes em éguas Mangalarga Marchador suplementadas com Saccharomyces cerevisiae durante treinamento aeróbico ${ }^{1}$
}

\section{Digestibility and nutrient intake in Mangalarga Marchador mares supplemented with Saccharomyces cerevisiae during aerobic training}

\author{
Tiago Resende Garcia²; Adalgiza Souza Carneiro de Rezende ${ }^{3 *}$; \\ Juliano Martins Santiago ${ }^{2}$; Rosângela Antunes Terra²; \\ Mayara Gonçalves Fonseca²; Maria Lindomárcia Leonardo da Costa²; \\ Ângela Maria Quintão Lana ${ }^{3}$; Fernando Queiroz de Almeida ${ }^{4}$
}

\section{Resumo}

\begin{abstract}
O trabalho avaliou o efeito da suplementação com leveduras sobre a digestibilidade e consumo dos nutrientes de equinos Mangalarga Marchador submetidas a treinamento. Quatorze éguas Mangalarga Marchador foram distribuídos em dois grupos: Probiótico (equinos suplementados com $20 \mathrm{~g}$ de Saccharomyces cerevisiae diariamente) e Controle. A dieta foi composta por volumoso e concentrado comercial, na proporção de 50:50. As éguas foram treinadas durante seis semanas, de segunda a sábado, sendo o exercício realizado diariamente alternando o trabalho em esteira ergométrica e caminhador automático. A digestibilidade dos nutrientes foi avaliada utilizando o indicador LIPE $^{\circledR}$ (6 dias) e a coleta de fezes foi realizada durante cinco dias. Foi analisado MS, FDN, FDA, PB, EB, hemicelulose e consumo de matéria seca. Não houve diferença $(P>0,05)$ em nenhuma das variáveis analisadas. A suplementação com $20 \mathrm{~g}$ de Saccharomyces cerevisiae não interfere na digestibilidade e consumo dos nutrientes em éguas Mangalarga Marchador submetidas a seis semanas de treinamento aeróbico.
\end{abstract}

Palavras-chave: Equino, fibra, hemicelulose, levedura, probiótico

\begin{abstract}
The study evaluated the effect of yeast supplementation on the digestibility and intake of nutrients of Mangalarga Marchador horses in training. Fourteen Mangalarga Marchador mares were divided into two groups: Probiotic (horses supplemented with $20 \mathrm{~g}$ of Saccharomyces cerevisiae daily) and Control. The diet consisted of commercial concentrate and roughage in the ratio of 50:50. The mares were trained for six weeks, Monday to Saturday, and the exercise performed daily alternating work on a treadmill and automatic walker. Nutrient digestibility was assessed using the indicator LIPE $^{\circledR}$ (6 days) end fecal collection was performed for five days. Was analyzed DM, NDF, ADF, CP, GE, hemicelluloses and dry matter intake. There was not difference $(P>0,05)$ in any of the variables analyzed. Supplementation with $20 \mathrm{~g}$ of Saccharomyces cerevisiae does not affect the digestibility and nutrient intake in mares Mangalarga Marchador submitted six weeks of aerobic training.
\end{abstract}

Key words: Equine, fiber, hemicellulose, probiotic, yeast

\footnotetext{
${ }^{1}$ Parte integrante da Tese de Doutorado do primeiro autor.

${ }^{2}$ Discentes do Programa de Pós-Graduação em Zootecnia, Universidade Federal de Minas Gerais, UFMG, Belo Horizonte, MG. E-mail: tirega@gmail.com; jmartinssantiago@yahoo.com.br; roveterinaria@hotmail.com; mayaragoncalvesf@hotmail.com; lindomarciacosta@gmail.com

${ }^{3}$ Profs. Associados, Dept ${ }^{\circ}$ de Zootecnia, UFMG, Belo Horizonte, MG. E-mail: adalgizavetufmg@gmail.com; angelaquintao@ gmail.com

${ }^{4}$ Prof. Associado, Instituto de Veterinária, Universidade Federal Rural do Rio de Janeiro, UFRRJ, Belo Horizonte, MG. E-mail: almeidafq@yahoo.com.br

* Autor para correspondência
} 


\section{Introdução}

Os probióticos são microrganismos vivos que conferem benefícios à saúde do hospedeiro por regular a atividade intestinal, restaurando sua microbiota natural (JULLIAND, 2006). Nos animais submetidos a estresse, a suplementação com probióticos tem como objetivo restabelecer o equilíbrio entre os microrganismos intestinais, aumentando a resistência às doenças e evitando a perda de peso. Além disso, nos equinos a garantia da qualidade da microbiota do ceco-cólon, através da suplementação com probióticos, pode otimizar a digestibilidade dos nutrientes da dieta, especialmente dos componentes fibrosos (MORGAN et al., 2007; REZENDE et al., 2012). De acordo com Julliand (2006), a digestibilidade da matéria seca (MS), fibra em detergente neutro (FDN), fibra em detergente ácido (FDA), proteína bruta (PB), energia bruta (EB) e hemicelulose da dieta varia com a cepa do microrganismo, a quantidade suplementada e a composição da dieta.

Kim et al. (1991) avaliando a suplementação de equinos adultos com Saccharomyces cerevisiae observaram aumento $(\mathrm{P}<0,05)$ na digestibilidade da MS, FDN, FDA e hemicelulose. Moore et al. (1994) relataram aumento $(\mathrm{P}<0,05)$ da digestibilidade da MS, FDN, FDA e da PB, ao adicionar $10 \mathrm{~g}$ de leveduras na dieta de pôneis. Resultado semelhante foi registrado por Hill et al. (2001), com aumento na digestibilidade aparente da $\mathrm{PB}$, de 64,3 para $72,8 \%$. Além do aumento na digestibilidade da FDN, Hill e Gutsell (1998) também relataram maior absorção intestinal do fósforo quando suplementaram equinos com probióticos.

Em potros, Hausenblasz, Szuco e Mezes (1993) observaram que a suplementação diária com levedura $(8 \mathrm{~g})$ aumentou $(\mathrm{P}<0,05)$ a digestibilidade da MS e PB. Glade e Sist (1988) forneceram menores concentrações de leveduras (4 g) para potros aos 12 meses de idade e relataram aumento $(\mathrm{P}<0,05)$ na digestibilidade da MS, FDN e FDA, de $68,6 \% ; 51,7 \%$ e $51,2 \%$ para $73,4 \% ; 60,7 \%$ e $58,2 \%$, respectivamente. Embora não tenha observado efeito da suplementação de potros com $30 \mathrm{~g}$ de leveduras na digestibilidade da fração fibrosa da dieta, Ribeiro (1998) registrou aumento $(\mathrm{P}<0,05)$ na digestibilidade da $\mathrm{PB}$, de 70,9 para $74,5 \%$, no grupo suplementado. Segundo Moura et al. (2009), os probióticos podem ser utilizados como estratégia nutricional para melhorar o aproveitamento da dieta em potros desmamados, pois as leveduras aumentam a utilização do componente mais digestível da fibra, a hemicelulose.

Em relação à qualidade da forragem, Morgan et al. (2007) avaliando a suplementação de equinos com Saccharomyces cerevisiae (56 g/animal/dia) sobre o aproveitamento de forrageiras de alta e baixa qualidade, relataram aumento $(\mathrm{P}<0,05)$ na digestibilidade de FDN e PB somente no grupo que consumiu forrageiras de baixa qualidade. Furtado et al. (2010), avaliando a suplementação de equinos com Saccharomyces cerevisiae também observaram que a qualidade nutricional do feno influenciou os coeficientes de digestibilidade do extrato etéreo (EE). Além do aumento na digestibilidade de FDN, Agazzi et al. (2011) também registraram maior digestibilidade aparente da MS e FDA em equinos adultos consumindo dieta com alto teor de fibra e suplementados com levedura. Já Lattimer et al. (2007) testando o efeito da suplementação com leveduras em equinos consumindo dietas com alta proporção de fibra ou alta proporção de concentrado observaram aumento $(\mathrm{p}<0,05)$ somente na produção de acetato, nas dietas com alta proporção de concentrado.

Rezende et al. (2012) avaliando o efeito da suplementação com $20 \mathrm{~g}$ de probióticos em equinos submetidos a treinamento, observaram aumento da digestibilidade da hemicelulose no grupo suplementado $(\mathrm{P}<0,05)$ da energia digestível (ED). Segundo estes autores, os aditivos probióticos podem ser adicionados na dieta de equinos em treinamento, visando aumentar a digestibilidade da fração fibrosa e ED da dieta, a fim de incrementar o aporte energético dos animais. 
O presente estudo teve como objetivo avaliar os efeitos da suplementação com Saccharomyces cerevisiae na digestibilidade da MS, FDN, FDA, $\mathrm{PB}, \mathrm{EB}$, hemicelulose e consumo de matéria seca em éguas da raça Mangalarga Marchador, submetidas a seis semanas de treinamento aeróbico em esteira ergométrica de alta velocidade e caminhador automático.

\section{Material e Métodos}

Foram utilizadas 14 éguas da raça Mangalarga Marchador não gestantes, com idades entre quatro e nove anos e com peso corporal entre 320 e 413 $\mathrm{kg}$. O delineamento experimental foi inteiramente ao acaso com dois tratamentos (grupo Probiótico e grupo Controle), com sete repetições por tratamento.

Os animais foram alojados em baias individuais de alvenaria, com comedouros separados para feno e concentrado e bebedouros para livre acesso à água. Os equinos foram alimentados com dieta composta por feno de Coastcross (Cynodon dactylon) e concentrado comercial (Expander Atleta Itambé ${ }^{\circledR}$ ) sem adição de leveduras. No início do período experimental e semanalmente as éguas foram pesadas. A cada pesagem, a quantidade de alimento a ser fornecida diariamente foi calculada como $2,5 \%$ do peso vivo de cada animal, o que representou o consumo de MS até a próxima pesagem. A quantidade de concentrado fornecida representou $50 \%$ desse valor. Esses valores foram determinados para equinos em trabalho moderado, de acordo com o NRC (2007). O alimento concentrado foi dividido em três porções iguais diárias, fornecidas às 6,12 e 18 horas, e o feno em duas porções iguais, as 8 e 19 horas. $\mathrm{O}$ sal mineralizado foi oferecido à vontade.

$\mathrm{O}$ experimento foi dividido em duas fases. $\mathrm{Na}$ primeira fase, com duração de 15 dias, as éguas foram adaptadas às instalações, manejo e ao exercício em esteira ergométrica de alta velocidade (Galloper Sahinco ${ }^{\circledR}$ ) e caminhador automático $\left(\right.$ Sahinco $\left.{ }^{\circledR}\right)$. Na segunda fase, com duração de 42 dias, as éguas foram submetidas a treinamento aeróbico, e o grupo
Probiótico passou a receber diariamente, além da dieta padrão, $20 \mathrm{~g}$ de Saccharomyces cerevisiae (Lesaffre Feed Additives - Biosaf Sc $47^{\circledR}$ ) (mínimo de 1,0 x $109 \mathrm{UFC} / \mathrm{g}$ ), juntamente com o primeiro fornecimento de concentrado.

As éguas foram treinadas durante seis semanas, de segunda a sábado, alternando o trabalho na esteira ergométrica e no caminhador automático. Sete animais treinaram na esteira segunda, quarta e sexta-feira e sete na terça, quinta e sábado. Nos dias em que não trabalharam na esteira, as éguas eram exercitadas ao passo no caminhador e no domingo nenhum animal foi submetido aos trabalhos.

$\mathrm{O}$ protocolo de treinamento na esteira ergométrica consistiu de um período inicial de aquecimento de 10 minutos (min.), sendo $5 \mathrm{~min}$. ao passo na velocidade de 1,6 metros por segundo $(\mathrm{m} / \mathrm{s})$ e $5 \mathrm{~min}$. na marcha a $3,5 \mathrm{~m} / \mathrm{s}$. Em seguida, a esteira foi inclinada a $3^{\circ}$ e realizados $30 \mathrm{~min}$. de marcha em velocidade específica individual. Essa velocidade correspondeu a $80 \%$ da velocidade do limiar aeróbico de cada animal, determinada em um teste padrão de esforço máximo com uso de máscara espirométrica, e variou de 3,6 a $4,4 \mathrm{~m} / \mathrm{s}$ entre os animais. $\mathrm{O}$ desaquecimento foi realizado durante $10 \mathrm{~min}$., sendo $5 \mathrm{~min}$. na marcha $(3,5 \mathrm{~m} / \mathrm{s})$ e $5 \mathrm{~min}$. ao passo $(1,6 \mathrm{~m} / \mathrm{s})$, sem inclinação da esteira. O exercício no caminhador consistiu de $60 \mathrm{~min}$. ao passo na velocidade de $1 \mathrm{~m} / \mathrm{s}$, sendo $30 \mathrm{~min}$. de rotação no sentido horário e $30 \mathrm{~min}$. no sentido antihorário.

Nos seis últimos dias da segunda fase experimental, foi fornecido as éguas, juntamente com a porção de concentrado das 12 horas, o indicador externo de digestibilidade lignina purificada e enriquecida (LIPE ${ }^{\circledR}$ ), na dose de $500 \mathrm{mg} /$ animal/ dia (LANZETTA et al., 2009). Para a adequada adaptação dos animais e eliminação uniforme do indicador nas fezes, o seu fornecimento foi iniciado um dia antes de começar a coleta das amostras de fezes, que foi feita diretamente na ampola retal, no período da tarde, uma vez ao dia, nos cinco 
dias subsequentes. As amostras de fezes foram acondicionadas em sacos plásticos, identificadas e imediatamente congeladas em freezer até seu processamento.

Amostras de forragem e concentrado foram coletadas no início, meio e final do período experimental, para avaliação da composição bromatológica e da digestibilidade in vitro da matéria seca (DIVMS). Cada uma dessas amostras também foi acondicionada em saco plástico, identificadas e congeladas em freezer para posteriores análises.

As amostras de fezes e dos alimentos concentrado e volumoso foram descongeladas à temperatura ambiente. Para cada animal foi realizado um pool das fezes referente aos cinco dias de coleta, com retirada de uma amostra com aproximadamente 400 g. O material obtido desse pool foi pesado e préseco em estufa de ventilação forçada a $65^{\circ} \mathrm{C}$ durante 72 horas. Após secagem, foram pesadas e moídas em moinho tipo Willey $\left(\right.$ Retsch $\left.^{\circledR}\right)$ com peneira de
$1 \mathrm{~mm}$ e acondicionados em frascos de polietileno hermeticamente fechados, para posterior análise de produção fecal e para as análises bromatológicas. As amostras de concentrado e volumoso, seguindo a mesma metodologia realizada para as amostras de fezes, também foram secadas, moídas e acondicionadas para análises de sua composição bromatológica e DIVMS. Foram determinados os teores de matéria seca (MS), proteína bruta (PB), energia bruta $(\mathrm{EB})$, matéria mineral $(\mathrm{MM})$, cálcio (Ca) e fósforo $(\mathrm{P})$, de acordo com o método da AOAC (1980). Os teores de fibra em detergente neutro (FDN), fibra em detergente ácido (FDA) e lignina foram obtidos de acordo com o método de Van Soest, Robertson e Lewis (1991). Para obtenção da digestibilidade dos nutrientes do volumoso e concentrado, realizou-se a técnica de DIVMS, seguindo a metodologia de Tilley e Terry (1963). Os valores da análise bromatológica e da DIVMS dos nutrientes componentes da dieta estão representados na tabela 1 .

Tabela 1. Percentual de matéria seca (MS), proteína bruta (PB), fibra em detergente neutro (FDN), fibra em detergente ácido (FDA), lignina (LIG), matéria mineral (MM), cálcio (Ca), fósforo $(\mathrm{P})$, energia bruta (EB) e digestibilidade in vitro da matéria seca (DIVMS) dos alimentos concentrado (C) e volumoso (V) fornecidos em diferentes momentos do período experimental (I: início; II: meio e III: final).

\begin{tabular}{lcccccccccc}
\hline Alimento & $\begin{array}{c}\text { MS } \\
(\%)\end{array}$ & $\begin{array}{c}\text { PB } \\
(\%)\end{array}$ & $\begin{array}{c}\text { FDN } \\
(\%)\end{array}$ & $\begin{array}{c}\text { FDA } \\
(\%)\end{array}$ & $\begin{array}{c}\text { LIG } \\
(\%)\end{array}$ & $\begin{array}{c}\text { MM } \\
(\%)\end{array}$ & $\begin{array}{c}\text { Ca } \\
(\%)\end{array}$ & $\begin{array}{c}\text { P } \\
(\%)\end{array}$ & $\begin{array}{c}\text { EB } \\
(\mathrm{Mcal} / \mathrm{kg})\end{array}$ & $\begin{array}{c}\text { DIVMS } \\
(\%)\end{array}$ \\
\cline { 2 - 10 } C I & 78,57 & 17,97 & 32,69 & 10,28 & 2,67 & 8,35 & 1,17 & 1,15 & 4,54 & 83,51 \\
C II & 82,24 & 18,5 & 31,90 & 9,51 & 2,29 & 8,33 & 1,14 & 1,10 & 4,29 & 86,09 \\
C III & 90,23 & 16,95 & 29,40 & 8,77 & 2,19 & 7,13 & 1,08 & 1,00 & 4,20 & 88,38 \\
Média & 83,68 & 17,81 & 31,33 & 9,52 & 2,38 & 7,94 & 1,13 & 1,08 & 4,34 & 85,99 \\
\hline V I & 89,26 & 5,33 & 85,33 & 40,43 & 4,84 & 8,74 & 0,54 & 0,34 & 4,18 & 42,19 \\
V II & 90,84 & 5,28 & 83,41 & 39,35 & 4,98 & 7,58 & 0,48 & 0,27 & 4,23 & 43,33 \\
V III & 91,62 & 6,39 & 85,68 & 40,53 & 5,00 & 4,75 & 0,28 & 0,30 & 4,38 & 42,43 \\
Média & 90,57 & 5,67 & 84,81 & 40,10 & 4,94 & 7,02 & 0,43 & 0,30 & 4,26 & 42,65 \\
\hline
\end{tabular}

Fonte: Elaboração dos autores.

A técnica empregada para análise do teor do indicador LIPE $^{\circledR}$ nas fezes foi a espectroscopia de infravermelho, utilizando o equipamento FTIVLAB com HATR (Búler ${ }^{\circledR}$ ). O teor de LIPE $^{\circledR}$ foi determinado nas fezes, estimando-se a produção fecal. Os valores de produção fecal foram calculados conforme descrito por Lanzetta et al. (2009):

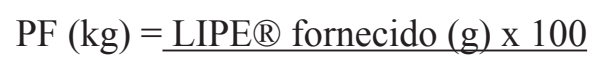

(Ai / MStotal ) 
Onde:

$\mathrm{PF}=$ Produção fecal; Ai = Relação logarítmica das intensidades de absorção das bandas dos comprimentos de onda a $1050 \mathrm{~cm}-1 / 1650 \mathrm{~cm}-$ 1; $\mathrm{MS}$ total = matéria seca fecal total; $\mathrm{O}$ Ai foi calculado através da fórmula: $\mathrm{Ai}=\mathrm{A} 1050$ / A1650; sendo que: $\mathrm{A}=\log \mathrm{I} 0$

Onde: I0 > intensidade e I $<$ intensidade.

O coeficiente de digestibilidade aparente (CDA) foi obtido conforme o estimador:

$\operatorname{CDA}(\%)=\frac{(\text { Nutriente ingerido })-(\text { Nutriente excretado nas fezes }) \times 100}{(\text { Nutriente ingerido })}$

Com os valores de produção fecal $(\mathrm{PF})$ e da digestibilidade in vitro, estimou-se o consumo de matéria seca (CMSLIPE) da forrageira fornecida aos animais, conforme o estimador:

$$
\text { CMSLIPE }=\frac{\text { PF }}{(1-\text { DIVMS } / 100)}
$$

Onde:

CMS =Consumo de Matéria Seca; PF = Produção fecal; DIVMS= Digestibilidade in vitro da Matéria Seca
O resultados foram submetidos a análise de variância e as médias comparadas pelo teste de Fisher a 5\% de probabilidade, utilizando o software SAEG versão 9.0 (2007).

\section{Resultados e Discussão}

Não houve diferença $(\mathrm{P}>0,05)$ nos coeficientes de digestibilidade aparente de MS, FDN, FDA, $\mathrm{PB}, \mathrm{EB}$ e hemicelulose da dieta e no consumo de matéria seca, entre os grupos Probiótico e Controle, conforme demonstrado na tabela 2 .

Analisando vários estudos que testaram os efeitos da suplementação com Saccharomyces cerevisiae na digestibilidade da dieta, observa-se que os resultados são contraditórios. Em alguns casos, a suplementação com a levedura pode melhorar a digestibilidade de apenas um ou mais nutrientes da dieta (NRC, 2007). Isso pode estar relacionado à utilização de animais de diferentes idades, raças e sexo e diferentes quantidades de leveduras oferecidas, formulações de dietas e qualidade dos ingredientes (FURTADO et al., 2010). O sucesso dos efeitos dos probióticos depende também da cepa do microrganismo ofertada aos animais (JULLIAND, 2006; NRC, 2007).

Tabela 2. Médias dos coeficientes de digestibilidade aparente da matéria seca (MS), fibra em detergente neutro (FDN), fibra em detergente ácido (FDA), hemicelulose (HEM), proteína bruta (PB) e energia bruta (EB) e do consumo de matéria seca (CMS) dos equinos dos grupos Controle e Probiótico.

\begin{tabular}{|c|c|c|c|c|c|c|c|}
\hline \multirow{2}{*}{ Tratamento } & \multicolumn{6}{|c|}{ Coeficiente de digestibilidade aparente (\%) } & \multirow[b]{2}{*}{ CMS (kg/dia) } \\
\hline & MS & FDN & FDA & HEM & $\mathrm{PB}$ & EB & \\
\hline Controle & 65,5 & 61,3 & 55,9 & 65,3 & 66,5 & 63,6 & 8,2 \\
\hline Probiótico & 65,4 & 59,9 & 53,5 & 64,7 & 70,6 & 64,3 & 8,0 \\
\hline $\mathrm{CV}(\%)$ & 0,3 & 3,1 & 5,5 & 2,1 & 7,0 & 1,1 & 9,7 \\
\hline
\end{tabular}

Não houve diferença pelo teste de Fisher $(\mathrm{P}>0,05)$.

Fonte: Elaboração dos autores.

No presente estudo, a dieta oferecida aos animais foi balanceada e forneceu os nutrientes necessários para equinos em trabalho de intensidade moderada (NRC, 2007), respeitando a proporção de 50:50 de volumoso:concentrado. Cavalos em treinamento são alimentados com dietas com menor teor de fibra e ricas em amido, a fim de suprir suas necessidades energéticas (NRC, 2007). De acordo com Julliand (2006), a alta concentração de amido na dieta pode ultrapassar a capacidade de hidrólise do intestino 
delgado, permitindo a chegada de partículas não digeridas no intestino grosso, as quais são fermentadas. A rápida fermentação do amido no intestino grosso altera a composição da microbiota (JULLIAND et al., 2001), com proliferação de lactobacilos e estreptococos (GOODSON et al., 1988), levando a produção elevada de ácido lático e de outros ácidos orgânicos no intestino grosso (OWENS et al., 1998). Isso reduz o pH, dificultando a digestão da fibra (PAGAN, 1998; JULLIAND et al., 2001), além de aumentar o potencial de produção de endotoxinas pela lise das bactérias gram negativas (CLARKE; ROBERTS; ARGENZIO, 1990).

Medina, Jacotot e Julliand (2002), observaram concentrações reduzidas de bactérias celulolíticas e maiores concentrações de estreptococos, lactobacilos e bactérias que utilizam lactato em animais que receberam dietas com alto teor de amido e que foram suplementados com Saccharomyces cerevisiae, quando comparados aos que receberam dietas com alto teor de fibra. Nesse trabalho, os autores destacaram que a cepa do probiótico utilizada chegou ao ceco e ao cólon ventral direito e sobreviveu, mas não foi capaz de colonizá-los. De modo semelhante, no presente estudo o maior teor de amido da dieta pode ter diminuído as concentrações de bactérias celulolíticas no cecocólon, interferindo na digestibilidade da fibra. A colonização do intestino grosso pela levedura pode também não ter ocorrido.

Outrapossível causa da ausência de diferençaentre os tratamentos pode ser explicada pelo curto período em que os animais receberam a suplementação. As éguas do grupo suplementado com probióticos ingeriram a levedura Saccharomyces cerevisiae, por um período de seis semanas. Segundo Ward et al. (2004), quanto maior o tempo de utilização desses aditivos, mais significativos serão seus efeitos. No entanto, Rezende et al. (2012) também treinaram equinos durante 42 dias e verificaram resposta positiva dos probióticos.
Outro fator a ser considerado é o tipo de probiótico utilizado. Moura et al. (2009) comprovavam que o fornecimento de diferentes tipos de probióticos provocou diferenças $(\mathrm{P}<0,05)$ na digestibilidade dos ingredientes da dieta em equinos, dependendo do tipo da cepa oferecida. Os autores observaram aumento na digestibilidade somente da hemicelulose em potros suplementados com $5 \mathrm{~g} /$ dia da levedura viva Saccharomyces cerevisiae (mínimo de 1,0 x 109 UFC/g), afirmando que a suplementação com essa levedura poderia ter favorecido a população de bactérias celulolíticas no intestino grosso. Já no grupo suplementado com $2 \mathrm{~g}$ de bactérias + levedura (mínimo de 3,33 x $107 \mathrm{UFC/g}$ de Bifidobacterium bifidum; 1,66 x 107 UFC/g de Enterococcus faecium; 3,33 x $107 \mathrm{UFC} / \mathrm{g}$ de Lactobacillus acidophilus; 1,66 x $107 \mathrm{UFC} / \mathrm{g}$ de Lactobacillus plantarum e 3,33 x 105 UFC de Saccharomyces cerevisiae) foi observado maior digestibilidade somente da PB, demonstrando a melhora da atividade proteolítica da microbiota intestinal pela adição de bactérias espécie-específicas, devido ao estímulo da reciclagem endógena do nitrogênio.

Poroutrolado, Swyersetal.(2008)nãoverificaram efeito da suplementação com microrganismos probióticos espécie-específicos na digestibilidade de nenhum dos componentes da dieta de equinos. Os autores utilizaram cepas de Lactobacillus acidophilus em um grupo e uma composição de Lactobacillus acidophilus, Lactobacillus casei, Bifidobacterium bifidum e Enterococcus faecium em outro grupo, não observando diferença entre eles. Os autores também não observaram efeito do probiótico na redução do risco de acidose, pela suplementação com altas doses de amido na dieta.

No presente estudo, o resultado encontrado para consumo de matéria seca (CMS) confirma os achados de Morgan et al. (2007), que não observaram alteração no CMS em cavalos adultos suplementados com Saccharomyces cerevisiae. 


\section{Conclusões}

A suplementação com $20 \mathrm{~g}$ de Saccharomyces cerevisiae não interfere na digestibilidade da MS, FDN, FDA, PB, EB, hemicelulose e consumo de matéria seca em éguas Mangalarga Marchador submetidas a seis semanas de treinamento aeróbico.

Os procedimentos experimentais foram avaliados e certificados pelo Comitê de ética em experimentação animal da Universidade Federal de Minas Gerais (CETEA- UFMG) sob protocolo $n^{\circ}$ 206/2008.

\section{Referências}

AGAZZI, A.; FERRONI, M.; FANELLI, A.; MARACCOLO, S.; INVERNIZZI, G.; DELL'ORTTO, V.; SAVOINI, G. Evaluation of the effects of live yeast supplementation on apparent digestibility of High-Fiber diet in mature horse using the acid insoluble ash marker modified method. Journal of Equine Veterinary Science, New York, v. 31, n. 1, p. 13-18, 2011.

ASSOCIATION OFFICIALANALYTICALCHEMISTS INTERNATIONAL - AOAC. Official methods of analisys. 13. ed. Washington: AOAC, 1980. 1015 p.

CLARKE, L. L.; ROBERTS, M. C.; ARGENZIO, R. A. Feeding and digestive problems in horses: Physiologic responses to a concentrated meal. Veterinary Clinics of North America, Philadelphia, v. 6, n. 2, p. 433-450, 1990.

FURTADO, C. E.; BARBOZA, E. D.; BRANDI, R. A.; RIBEIRO, L. B.; OLIVEIRA, A, A, M, A. Uso de levedura em equinos alimentados com dietas compostas de fenos de diferentes qualidades nutricionais. Revista Brasileiro de Zootecnia, Viçosa, v. 39, n. 10, p. 21942199, 2010.

GLADE, M. J.; SIST, M. D. Dietary yeast culture supplementation enhances urea recycling in the equine large intestine. Nutrition Reports International, Washington, v. 37, n. 1, p. 11-17, 1988.

GOODSON, J.; TYZNIK, W. J.; CLINE, J. H.; DEHORITY, B. A. Effects of an abrupt diet change from hay to concentrate on microbial numbers and physical environment in the cecum of the pony. Applied and Environmental Microbiology, Washington, v. 54, n. 8, p. 1946-1950, 1988.

HAUSENBLASZ, J.; SZUCO, J.; MEZES, M. Effect of viable yeast culture. Supplementation on nutrient digestibility and feed utilization of growing cold-blooded horses. In: PROCEEDINGS OF ALLTECH'S ANNUAL SYMPOSIUM, 9., 1993, Nottingham. Proceedings... Nottingham: Nottingham University Press, 1993. p. 4552.

HILL, J.; GUTSELL, S. Effect of supplementation of a hay and concentrate diet with live yeast culture on the digestibility of nutrients in 2 and 3 years old riding school horses. In: BRITISH SOCIETY OF ANIMAL SCIENCE, 1998, Medlothian. Proceedings... Medlothian: British Society of Animal Science, 1998. p. 128.

HILL, J.; TRACEY, S. V.; WILLIS, M.; JONES, L.; ELLIS, A. D. Yeast culture in equine nutrition and physiology. Science and technology in the feed industry. In: PROCEEDINGS OF ALLTECH'S ANNUAL SYMPOSIUM, 17., 2001, Nottingham. Proceedings... Nottingham: Nottingham University Press, 2001. p. $97-$ 114.

JULLIAND, V. Pre- and probiotics: potentials for equine practice. In: EUROPEAN EQUINE NUTRITION AND HEALTH CONGRESS, 3., 2006, Merelbeke. Proceedings... Merelbeke: Ghent University, 2006. p. 17-18.

JULLIAND, V.; DE FOMBELLE, A.; DROGOUL, C.; JACOTOT, E. Feeding and microbial disorders in horses: 3 - effects of three hay: grain ratios on microbial profile and activities. Journal of Equine Veterinary Science, New York, v. 21, n. 11, p. 543-546, 2001.

KIM, S. M.; KIM, C. M.; LEE, H. K.; PARK, W.; LIM, P. Y.; KIM, J. B. J.; CHUNG, T. Y. Evaluation of nutrients values of some feedstuffs, and the effects of yeast culture supplementation on digestibilities of nutrients and blood parameter in horse. Korean Journal of Animal Nutrition and Feeds, Seoul, v. 15, n. 5, p. 272-280, 1991.

LANZETTA, V. A. S.; REZENDE, A. S. C.; SALIBA, E. O. S.; LANA, A. M. Q.; RODRIGUEZ, N. M.; MOSS, P. C. B. Validação do LIPE como método para determinar a digestibilidade dos nutrientes em equinos. Revista Brasileira de Zootecnia, Viçosa, v. 38, n. 1, p. 69-74, 2009.

LATTIMER, J. M.; COOPER, S. R.; FREEMAN, D. W.; LALMAN, D. L. Effect of yeast culture on in vitro fermentation of a high-concentrate or high-fiber diet using equine fecal inoculum in a daisy II incubator. Journal of Animal Science, Champaign, v. 85, n. 10, p. 2484-2491, 2007.

MEDINA, B.; JACOTOT, E.; JULLIAND, V. Effect of a preparation of Saccharomyces cerevisiae on microbial profiles and fermentation patterns in the large intestine of horses fed a high fiber or a high starch diet. Journal of Animal Science, Champaign, v. 80, n. 10, p. 2600-2609, 2002. 
MOORE, B. E.; NEWMAN, K. E.; SPRING, P.; CHANDLER, V. E. The effect of yeast culture (YeaSacc1026) in microbial populations and digestion in the cecum and colon of the equine. Journal of American Science, Champaign, v. 72, p. 252, 1994. Supplement 1.

MORGAN, L. M.; COVERDALE, J. A.; FROETSCHEL, M. A.; YOON, I. Effect of yeast culture supplementation on digestibility of varying forage quality in mature horses. Journal of Equine Veterinary Science, New York, v. 27, n. 6, p. 260-265, 2007.

MOURA, R. S.; SALIBA, E. O. S.; ALMEIDA, F.Q.; LANA, A. M. Q.; SILVA, V. P. S.; REZENDE, A. S. C. Feed efficiency in Mangalarga Marchador foals fed diet supplemented with probiotics of phytase. Revista Brasileira de Zootecnia, Viçosa, v. 38, n. 6, p. 10451050, 2009.

NUTRIENT REQUIREMENTS OF HORSES - NRC. Washington: National Academy Press, 2007. 341 p.

OWENS, F. N.; SECRIST, D. S.; HILL, W. J.; GILL, D. R. Acidosis in cattle: a review. Journal of Animal Science, Champaign, v. 76, n. 1, p. 275-286, 1998.

PAGAN, J. D. Carbohydrates in equine nutrition. In: PAGAN, J. D. (Ed.). Advances in equine nutrition. Nottingham: Nottingham University Press, 1998. p. 5770 .

REZENDE, A. S. C.; TRIGO, P.; LANA, A. M. Q.; SANTIAGO, J. M.; SILVA, P.; CASTEJON, F. M. Yeast as a feed additive for training horses. Ciência $e$ Agrotecnologia, Lavras, v. 36, n. 3, p. 354-362, 2012.
RIBEIRO, A. C. A. Efeitos da adição de cultura de levedura na digestibilidade de nutrientes para equinos. 1998. Dissertação (Mestrado em Zootecnia) Universidade de São Paulo. Faculdade de Zootecnia e Engenharia de Alimento, Pirassununga.

SISTEMAS PARA ANÁLISES ESTATÍSTICAS SAEG versão 9.0. Viçosa: Fundação Arthur Bernardes, 2007.

SWYERS, K. L.; BURK, A. O.; HARTSOCK, T. G.; UNGERFELD, E. M.; SHELTON, J. L. Effects of direct-fed microbial supplementation on digestibility and fermentation end-products in horses fed low- and high- starch concentrates. Journal of Animal Science, Champaign, v. 86, n. 10, p. 2596-2608, 2008.

TILLEY, J. M.; TERRY, R. A. A two-stage technique for the in vitro digestion of forage crops. Journal of the British Grassland Society, Oxford, v. 18, n. 2, p. 104-111, 1963.

VAN SOEST, P. J.; ROBERTSON, J. B.; LEWIS, B. A. Methods for dietary fiber, neutral detergent fiber, and nonstarch polyssacharides in relation to animal nutrition. Journal of Dairy Science, Champaign, v. 74, n. 10, p. 3583-3597, 1991.

WARD, M. P.; ALINOVI, C. A.; COUËTIL, L. L.; GLICKMAN, L. T.; WU, C. C. A randomized clinical trial using probiotics to prevent Salmonella fecal sheddings in hospitalized horses. Journal of Equine Veterinary Science, New York, v. 24, p. 242-247, 2004. 